\title{
Kansei engineering, emotional design and ergo design as shaping tools in architecture and design
}

\author{
Ilgina Shakirova ${ }^{1}$ [0000-0001-8805-4836] \\ ${ }^{1}$ Kazan State University of Architecture and Engineering, 420043, Zelenaya st., Kazan, Russia
}

\begin{abstract}
This article considers the shaping methods in architecture and design, namely emotional design and ergo design and Kansei engineering. The essence of these shaping methods explains how these methods relate to each other and how they interact at different levels. There is a comparison of these shaping methods and an explanation why they should develop in parallel. It describes why these sciences are interpreted as human-oriented methods of object-based shaping and how they force us to rethink the human approach to the perception to familiar objects, and also describes their influence on the perception of surrounding objects by humans. Each of the presented shaping methods is considered as a new branch affecting different human life aspects. Article considers the fundamental factors of shaping methods, their relevance in the modern world, both as in the industrial and in the research worlds. Methods can be viewed collectively and separately, since each of them is functional and independent.
\end{abstract}

Keywords: architecture, design, shaping, emotional design, ergo design, design objects, design theory.

\section{Introduction}

In our country, theoretical developments in the design field carried out only from the $60 \mathrm{~s}$ years of XX century, since creation the state design system in the country was headed by VNIITE [1].

An integral part of many interrelated scientific and methodological research areas was the work on the streamlining design theory problem by the conceptual and terminological apparatus of technical aesthetics [2].

In the XIX century and even at the beginning of the twentieth century in Englishspeaking countries, the word «design», given its polysemy, was widely used in relation to handicrafts, arts and crafts, graphic art, meaning «drawing», "pattern», «sketch», «composition». One of the books of English art theorist pre-Raphaelite ideologue John Ruskin (1819-1900) was called «Hints on Pattern designing». Famous English applied arts artist William Morris (1834-1896) was the «Arts and Crafts Movement» founder and often was called as s designer by his work researchers. The authors of the book «Modern History Applied Art» R. Rosenthal and H. Ratzka used the word «design» both in relation to applied art and design itself [3,4]. 
Necessary awareness to develop a design theory as a scientific discipline first began in the socialist countries: Czechoslovakia, Poland, the German Democratic Republic, and since 1962 in the USSR [5].

In 1954 the Czech designer and scientist P. Tuchny introduced the term «technical aesthetics». He outlined to the artistic design tools theory and production means, scientific methods of improving working conditions, in particular, improving the various tools shape based on physiological and anthropometric data [6].

In our country, this term was borrowed and also at first mistakenly associated with the concept of a general production culture (with « production aesthetics «). It began to denote, in fact, not the design theory, but the practice associated with raising the culture of production by artistic design means [7]. However, later the term «technical aesthetics» was critically rethought in our country and began to be used in a different meaning - the general theory of artistic and technical creativity in industry, that is design theory [8].

Technical aesthetics is the science of the artistic creation laws in the technology field, which it reveals by studying the relationship between a person and the material culture objects created in the environment where this relationship is carried out [9]. Therefore, technical aesthetics formulates requirements not only for various products created by means of industrial production, but also for the environment in which they are used and aesthetically perceived. This scientific discipline comprehensively studies the social, aesthetic, functional, ergonomic and technical and technological aspects of the subject environment formation in various human life spheres and creates the scientific and methodological design base $[10,11]$.

\section{Research methods}

The design activity subject is the creation of harmonious, meaningful and expressive object forms, which reflect the integral meaning of their use value.

Almost any technical industrial product (kit, ensemble, complex, system) in any sphere and human life environment, where human communication is socially and culturally conditioned (to one degree or another), can become as a design object [12].

Design is a specific project activity type that combines the artistic ideology subject creativity with engineering design practice focused on industrial production. Along with the existing design types (architectural, engineering), at the beginning of the twentieth century another design type was formed - artistic design and corresponding to it activity social institution called design $[13,14]$.

In design, the activity theoretical area subject is the nature comprehension, origin, purpose, objectives, design functions, its structure study, basic concepts, principles and patterns, the design methods development as a design and creative activity focused on humanizing the human life environment through its harmonization. The design activity subjects of this area are theorists and methodologists [15].

Changes have taken place in the modern world. Humanity's priorities and needs have changed. This also affected modern design, there was a certain design evolution, which ultimately led to the fact that today emotions and convenience came to the fore in design.

The desire to understand the human things world vision and its reflection in design, was created the philosophy of emotional design [16].

\section{Results and discussion}

Emotional design is called as the language of the heart. In contrast to rational, it creates a product that is able to «pick up» the consumer on the emotional hook and be a cause of 
certain feelings in person. But, according to Aaron Walter, the author of the term «emotional design», the product must meet the requirements of functionality, reliability and value, and only then affect the feelings. Only then consumer gets experience of positive emotions.

Emotional design is a deep concept. It unites the mass production objects formation and human emotions into a single whole. Emotions accompany us from the moment of birth all along the life path [17]. Emotions help us to express our attitude to objects around us, to actions and situations happening around us and inside us.

The design and experience optimization expert Aaron Walter in his book «Designing for Emotion» defines emotions «as the common language of humanity,» as a language that is familiar to anyone from the birth moment and is understood by people who speak different languages. Through emotions, we express our attitude to various objects: some things cause delight in us, some things cause disgust feelings, and still others «do not hurt a living,» and we are indifferent to them [18].

The term «Emotional Design» was first used by designer Aaron Walter. He describes this concept in his book Emotional Web Design, drawing an analogy with Maslow's pyramid of human needs. A renowned economist and sociologist, Maslow placed at the base of the pyramid the primary elementary physiological human needs for food, water, and clothing. On the next stage, he identified the needs of a person for security, housing [19]. And only through the satisfaction of their social needs for friendship, respect and public recognition, a person rises to the top of the pyramid - to the personal growth level and spiritual improvement.

Aaron Walter, constructing a pyramid to determine the product properties priority, based it on the product functionality, then - reliability, higher - convenience in its use, and at the top - the property of the product to give pleasure to the consumer [20]. That is, at the top of Walter's pyramid is what the emotional design purpose - to give the user pleasure, new positive impressions and emotions, because exactly emotions give the deepest imprint on a person's long-term memory. But the consumer will get emotional pleasure from using the product only after, according to Walter, that convinced of its functionality, reliability and convenience [21].

Donald Norman, American industrial design and perception psychology professor at the University of California, explains in his book Emotional Design (2004) why «attractive things work better» - because they create a sense of satisfaction by bringing to a minimum minor flaws from these things. If a person is satisfied, it is easier to think, easier to do.

Studying the brain work, Norman identified 3 levels of perception and information processing: intuitive, behavioral and reflex, correlating them with visual design.

At the intuitive level (visceral level), primary emotional reactions are manifested and instant decisions are made: like - dislike, good - bad, dangerous - safe. This is the unconscious level. The emotional processing of the product begins with it.

Behavioral (behavioral level) is also the unconscious level. It controls our behavior. We do any physical work: walk, swim, clean snow, push buttons, cook food. The goal of behavioral design is to give the user feeling that they control the situation. It manifested in the ease of understanding - how to use the object, its ease of use.

Reflex level (reflective level) is the "consciousness heart», its inner voice. It is the highest stage of development, which controls the conscious thinking process and higher emotions. At the reflex level of information processing, the product «picks up» the consumer by the «emotional hook» and causes a satisfaction feelings.

The impact duration on our emotional state of these each levels is different - the intuitive has the shortest. The reflex effect is the longest: the brain can return to thinking about the correctness of made decision, about the legitimacy of one or another committed act many times. The behavioral level of information processing leads us to a solution. And 
at the reflex level, the object, together with the accompanying factors, «clings to us», causes a satisfaction feeling.

Norman levels can act both individually and intertwined, flowing into one another. For example, we eat ice cream: the product affects our taste buds, we get pleasure (if the product is of high quality and we love it). Delicious food is the simplest and fastest joy in life, and our mood rises. As an example: milk in a bottle brought from a store tastes bitter. We determined it immediately, as soon as the bottle was opened and milk was tasted. We are upset. The intuitive level of product recognition worked for us. Let's develop the situation further - we went to return the bottle of spoiled milk to the store. Here the intuitive level flows smoothly into the behavioral one.

Norman Levels can be used as a guide to creating a product that is functional, reliable, usable, and attractive. But good product design involves a combination of these 3 levels in the right proportions.

Ergo design - is also among to such progressive technologies, which is a new type of design activity, different from the traditional ergonomic and artistic design. Ergo design as a scientific category is consistently established in the field of design and in the ergonomists circles, which is naturally reflected in the strengthening of its scientific and practical significance. Among specialists in the human factor research field, ergo design is interpreted as a human-oriented scientific and project activity, in which, due to the design and ergonomics integration means, aesthetically and ergonomically complete objects and a subject-spatial environment are created.

Another, relatively new, progressive technology for the complex design objects perception by a person is ergo design. Ergo design made itself known as an independent direction in the middle of the last century. Its origin is associated with the first international «Ergodesign» symposium in Switzerland in 1984. Ergo design arises from close interaction, penetration and interweaving of two independent scientific knowledge branches ergonomics and design, with its own methodology, scientific research principles, tools and ways to implement results.

Ergo design is interpreted as a human-oriented scientific and project activity, as a result of which an aesthetically and ergonomically valuable object-spatial environment is created for a person.

The efforts application vector of specialists in this field is aimed at the entire objective world that surrounds a person and consumed. With this definition of ergo design, we are talking about the entire object environment surrounding a person. One of the ergo design key tasks is improving the human life quality through the creation of convenient and comfortable working conditions.

The employee successful labor activity at the enterprise is not only an abundance of high-tech equipment and computer facilities, but also elementary convenience and reasonable organization of the workplace. They create the comfort that the employee feels throughout the working day. The idea of human labor as fundamental factor, in comparison with the machines and mechanisms activity, formed the basis scientific direction of the «ergo design», which are successfully being developed in many countries, including Russia.

For the first time the term «ergonomics» was used in 1857 by the Polish natural scientist Wojciech Jastrzębowski, that began to talk about ergonomics as a complex science at the beginning of the 20th century. At about the same time, the designers were seriously assigned the task of human work improving the conditions and quality, which formed the basis for ergo design appearing.

At the stages of its development, ergonomics performed its tasks in various industries: in the $50 \mathrm{~s}$ - in the military industry, in the $60 \mathrm{~s}$ - in industry, in the $70 \mathrm{~s}$ - in the consumer goods field, in the $80 \mathrm{~s}$ - in the work processes automation and computerization ergonomics, 
in the beginning of 21 century - the office environment ergonomics. Indeed, the ongoing workspace optimization and structures and parameters coordination of the subject work environment with the employee psychophysical characteristics turned out to be difficult tasks. To solve them, it was necessary to apply knowledge in the anthropology, biomechanics, psychology and physiology. For the human-machine system efficiently and safely cooperation, it is necessary to ensure maximum compatibility of both participants in the process. Anthropometric compatibility considers and takes into account the employee's body size, posture during work, compatibility from the sensorimotor view point - the speed of a person's motor operations and reaction to external stimuli - light, sound, etc. Biochemical compatibility is achieved by a balance of the employee's strength capabilities and the employee's efforts makes to control the equipment. Psychophysiological compatibility is a person's positive reaction to the aesthetic parameters of technology.

To create comfortable working conditions, ergo designers have to constantly study the relationship features between man and technology, improve the latter, periodically «adjusting» it to human capabilities. It is no coincidence that ergo design issues are under the close scrutiny of not only psychologists, physiologists, labor protection specialists, but also employers. Ergo design is recognized as a full-fledged scientific discipline that comprehensively studies a person in the specific conditions of activity in modern production. It resonates a wide response in the modern business environment, being as powerful interest integrator of employers and personal at the improving of working conditions and the workers life quality.

For almost four decades, Kansei engineering has been developing as an effective method of shaping, directly related to the industrial society and actively used in the postindustrial world. Many large companies have adopted Kansei engineering shaping techniques and created effective products. The method is most widely used in the automotive industry Kansei engineering, but this method is also actively used in other industries.

To have a more complete understanding of this shaping method, it is worth going back to the origins of this term. The term «Kansei» first appeared in the 17th century in Japanese philosophical literature. From the scientific point of view this term description occurred in 1878 by the Japanese explorer Nishi. He introduced this concept by investing in it the phrase definition «the ability to feel.» Also, the term «Kansei» was found in various works of other Japanese philosophers, but there it was used in the perception sense. Another Japanese philosopher Nishida uses the term «Kansei» in the «action intuition» concept where intuition implies action and acting intuition, that is, actions with an object are performed intuitively. According to his use of this term, one can understand that the world and objects are mutually formed and reflected in each other.

In the industrial design field, Kansei engineering appears towards the end of the twentieth century. At this time, a shift began to be observed in consumer behavior and their preferences became unexpected and logically different. Consumers in the objects choice became more unpredictable and their behavior began to resemble the term «Kansei», when the objects choice relied on the primary subject perception, it corresponds to the «Kansei» philosophy. That is, in this way, «Kansei» went beyond the scientific society and boldly stepped into the world of industry and society.

It becomes obvious that this is a single perception that provides a qualitative meaning and value of objects, this is a synthesis of emotional qualities from the visual object perception and its ergonomics in operation, respectively.

In general, the father of the term «Kansei engineering» is considered to be a professor Nagamachi at Hiroshima University. The term «Kansei engineering» was first used in 1986 by the president of the Mazda Automotive Corporation at the Michigan University. It is often said that Kansei engineering was first used in the design of Mazda Miata (also known 
as the MX-5). Sport cars must have a certain performance, but most importantly, they must be comfortable to drive. This is a classic example of finding a comfortable environment for humans and one the best cases which describes Kansei engineering.

Classical automotive engineering is primarily focused on manufacturing capabilities, performance and usability. At the same time Kansei engineering organizes its process based on providing the user with a targeted impression of the object. In the Mazda Miata case, this experience was characterized as Human Machine Unity: "This concept implies that the user feels the unity between driver and the car while driving. The driver feels that body can be a car and drive freely». Based on these considerations, the Kansei engineering approach has been structured to support the entire process towards the end result that allows the driver to experience this unity. From this, we can see that Kansei engineering is part of an engineering method aimed at transforming the user's feelings into specific product properties.

Kansei engineering does not develop new theories or tools in different areas. Rather, it is an overarching methodology containing rules for how different tools can interact with each other to quantify the impact of a particular product property on user experience. The tools come from other research areas (mathematics, computer science, psychology) and cooperate for Kansei engineering. Although the Kansei engineering structure remains stable, Kansei engineering can change dramatically when the tools involved in this method change. Accordingly, Kansei engineering can be described as a systems approach to shaping. Moreover, the Kansei engineering structure is a base, in which various tools and technologies can be attached.

Kansei engineering is defined by structure and purpose, tools and technology. However, Kansei engineering ignores all the characteristics of the Kansei method throughout the Kansei engineering shaping process. The objective approach that is required in shaping seems to contradict the subjective and predicative Kansei qualities. Therefore, it can be said that the Kansei engineering origin from Kansei was exaggerated. Kansei engineering does not aim to correlate directly the consumer response with design objects. Rather, Kansei engineering establishes a correlation between the user's expressed emotions and design objects.

The first scientific appearance of the term Kansei was in 1878, while Kansei engineering was first mentioned in 1986. Therefore, it is necessary to consider how the term Kansei developed before the Kansei engineering actual appearance, and correlate with the formation of physical objects: Kansei engineering and Kansei design.

Insofar as Kansei engineering is a symbiosis of shaping, based on design and sensory objects perception. That is, it is possible to trace the consumers cognitive processes associated with the preference and objects choice. We can say that Kansei engineering is a behavioral and psychological objects perception.

That is, human thinking and behavior in the design objects perception is based on psychology, but mainly on psychophysiology. Kansei engineering seeks to characterize and describe the emotional experience from the design objects perception, using knowledge gained on the basis of human physiology and psychology. Such an integrated approach to shaping helps to develop more and more new methods in subject shaping.

Based on Kansei engineering methods, the designer can collect a large store of knowledge based on the consumer perception. All this knowledge is obtained through the psychophysical perception prism of objects by a person.

One of the directions of Kansei engineering is presented in the form of Kansei design. Kansei engineering sources often use the term Kansei design to describe the Kansei engineering works that shape real-world objects. But, at upon closer viewing, it becomes clear that these works belong to the field of Kansei engineering, because they are still associated with an engineering approach to shaping, in contrast to Kansei design, which is 
just another approach. Kansei design projects can be divided into two groups. The first group focuses on the physical qualities of the object, i.e. on their intrinsic properties and on their rating or preference by the user. The second group is mainly devoted to the interactive objects essence, i.e. the object qualities in interaction. For example, an attempt at Kansei design developed by Toyota Motor Europe.

Originally, Kansei design was described as a way of introducing Kansei engineering approaches into the design thinking. The goal was to define a design area, create proposals that fit that space, and evaluate proposals based on consumer Kansei usage. Toyota Motor Europe used Kansei engineering methods for evaluation and came to understanding of the two-pronged design approaches necessity: understanding and creation, that is, felt the need for Kansei design. The Kansei design approach developed by Toyota Motor Europe aims to bring the user experience to the creation of design spaces.

While Kansei engineering is rooted in science-based approaches, more sophisticated sciences, while Kansei design tries to draw inspiration from earlier philosophical or cultural works, and use them as design inspiration. In addition, Kansei design encompasses the position of design as a guide to what we do, where we are going, and what catches the eye: Kansei design is people, our beliefs and our dreams, beauty and society. It's about being in the world.

There is a fundamental action principle: «We see a thing by action, and what we see defines us in the same way as we define a thing» this action is intuition. There is a fundamental body principle: «Just as the body of an artist is an organ of art.» The artist life exists in beauty, and the life of a scientist is in truth. Thinking does not exist apart from our physical body. This is what should be understood in design. It is necessary to consider Kansei design through action, and only then reflect on the action. Taking into account that western philosophers take similar position and are inspired by design approaches, Kansei design does not start from scratch, but can be associated with compatible ideas at the international, philosophical and intercultural level. Kansei design treats consumers with care and achieves fulfillment of needs through their respectful relationship between craftsmen and their objects. The object property is not revealed only by the consumers experience. It also reveals itself through the designer internationality. This is how Kansei design should be viewed in the shaping process, which includes all the viewpoints variety of designers and consumers.

In connection with the above, it is possible to correct or give another understanding of the term Kansei engineering and express it as an individual user experience from design items. Therefore, Kansei engineering is a shaping method focused on the object details consistency with the user's perception to identify and evaluate new design solutions.

\section{Conclusions}

Thus, we can conclude that emotional design is a first-order criterion, primary in relation to ergo design. And we tend to consider the convenience of using an object only after it «attracted» our attention emotionally. And ergo design is a second-order criterion, it is secondary in relation to emotional design, because if the object has not «hooked» us emotionally, we do not have a desire to get acquainted with its convenience and functionality. However, both of these technologies, complementing each other, help designers implement the main principles of human-centered design in their work. As for Kansei engineering, the situation here is quite different. When we consider an object from the point of emotional design view, the first thing we pay attention to is its aesthetic appearance, how pleasant the object is to us and whether we will continue to exploit it. In this case visual perception is primary and only then functionality. In ergo design, we consider objects from the point of view of functionality, paying attention to aesthetics at the 
last moment, or maybe not paying attention at all. That is, in ergo design, the object function, its operation convenience, ergonomics come to the fore, and the aesthetic object qualities are pushed into the background. But when we talk about Kansei engineering, we should understand the unity of aesthetics and function. In Kansei engineering, they do not rule over each other, but live in harmony forming harmonious objects of the human environment. Thanks to the shaping method Kansei engineering, a person lives in unity with the design objects and merges with them into a single whole, which makes it possible to exploit the design objects for pleasure.

\section{References}

1. S. D. Bernstein, Designs after nature: evolutionary fashions animals and gender, Victorian animal dreams: representations of animals in victorian literature and culture, ed Deborah Denenholz Morse and Martin A Danahay, London (2017) DOI: 10.4324/9781315235073-5

2. C. Buckley, The decorated object: gender, modernism and the design of industrial ceramics in britain in the 1930s: the gender of ornament, Women artists and the decorative arts 1880-1935, London, 53-72 (2019) DOI: 10.4324/9781315194486-4

3. E. Canli, Binary by design: Unfolding corporeal segregation at the intersection of gender, identity and materiality, Design Journal, 21(5), 651-669 (2018) DOI: 10.1080/14606925.2018.1491716

4. P. Meth, Informal housing, gender, crime and violence: the role of design in urban south Africa, The British Journal of Criminology, 57(2), 40-421 (2017) DOI: 10.1093/bjc/azv125

5. M. McIntyre Petersson, Gender by design: performativity and consumer packaging, Design and Culture, 10(3), 337-358 (2018) DOI: 10.1080/17547075.2018.1516437

6. S. Reimer, 'It's just a very male industry': gender and work in UK design agencies Gender Place and Culture A Journal of feminist geography, 23(7), 1033-1046 (2016) DOI: 10.1080/0966369X.2015.1073704

7. T. Christensen, J. Ball, Dimensions of creative evaluation: Distinct design and reasoning strategies for aesthetic, functional and originality judgments, Design Studies 45 (A), 116-136 (2016) DOI: 10.1016/j.destud.2015.12.005

8. N. Crilly, R. Morosanu, Creativity and fixation in the real world: Three case studies of invention, design and innovation, Design Studies, 64, 169-212 (2019) DOI: 10.1016/j.destud.2019.07.003

9. H. Casakin, V. Georgiev, Design creativity and the semantic analysis of conversations in the design studio, International Journal of Design Creativity and Innovation, 9, 61-77 (2021) DOI: 10.1080/21650349.2020.1838331

10. B. Emo, K. Al-Sayed, T. Varoudis, Design, cognition \& behaviour: usability in the built environment, International Journal of Design Creativity and Innovation, 4, 63-66 (2016) DOI: $10.1080 / 21650349.2016 .1143080$

11. J. Ventura, J. Bichard, Design anthropology or anthropological design? Towards «Social Design» International Journal of Design Creativity and Innovation 5 222-234 (2017) DOI: 10.1080/21650349.2016.1246205

12. J. Stein, Hidden Between Craft and Industry: Engineering Patternmakers Design Knowledge, Journal of Design History, 32(3), 280-303 (2019) DOI: 10.1093/jdh/epz012

13. M. Quinn, Design, History \& Time, Journal of Design History, 33(2), 189-191 (2020) DOI: $10.1093 / \mathrm{jdh} / \mathrm{epaa002}$

14. P. Garvey, A. Drazin, Design Dispersed: Design History, Design Practice and Anthropology, Journal of Design History, 29(1), 1-7 (2016) DOI: 10.1093/jdh/epv054 
15. K. Bonnevier, The Revue of STYLES, Architecture and Culture, 5(3), 353-369 (2017) DOI: $10.1080 / 20507828.2017 .1375342$

16. A. Shaouf, K. Lu, X. Li, The effect of web advertising visual design on online purchase intention: an examination across gender, Computers in Human Behavior, 60, 622-634 (2016) DOI: 10.1016/j.chb.2016.02.090

17. L. Busbea, Soft Control Material: Environment and Design c. 1970, Journal of Design History, 30(2), 139-156 (2017) DOI: 10.1093/jdh/epw033

18. A. Po , R. Holt, J. Wedgwood, Manufacturing and Craft, Journal of Design History, 29(2), 99-119 (2016) DOI: 10.1093/jdh/epv048

19. C. Guth, Crafting Community: George Nakashima and Modern Design in India, Journal of Design History, 29(4), 366-384 (2016) DOI: 10.1093/jdh/epw015

20. I. Lorenzo, The Role of Design Research in a Postindustrial Society, Design Principles and Practices: An International Journal - Annual Review, 13(1), 1-11 (2019) DOI:10.18848/1833-1874/CGP/v13i01/1-11.

21. I. Shakirova, Three waves of theoretical architecture and design, IOP Conference Series Materials Science and Engineering, 890, 012010 (2020) DOI: 10.1088/1757899X/890/1/012010 